\title{
On the representation of the electric charge distribution in ethane for calculations of the molecular quadrupole moment and intermolecular electrostatic energy
}

\author{
F. Y. Hansen and G. P. Alldredge ${ }^{\text {a) }}$ \\ Fysisk-Kemisk Institut, The Technical University of Denmark, DTH 206, DK 2800 Lyngby, Denmark \\ L. W. Bruch \\ Department of Physics, University of Wisconsin, Madison, Wisconsin 53706 \\ H. Taub \\ Department of Physics and Astronomy, University of Missouri-Columbia, Columbia, Missouri 65211
}

(Received 20 December 1984; accepted 19 March 1985)

\begin{abstract}
It is shown that a simple atom-site monopole model is inferior to one which includes higher-order local multipoles to represent the intramolecular charge distribution of ethane. Unlike the latter model, the local monopole representation predicts the wrong sign for the molecular quadrupole moment and gives a repulsive rather than an attractive electrostatic interaction at typical intermolecular distances. In the local multipole model, the atom-site dipoles give the largest contribution to both the molecular quadrupole moment and the intermolecular interaction.
\end{abstract}

Recently, by combining elastic neutron scattering ${ }^{1,2}$ and low-energy electron diffraction ${ }^{3}$ techniques, it has been possible to determine the structure of monolayer phases of ethane $\left(\mathrm{C}_{2} \mathrm{H}_{6}\right)$ adsorbed on graphite basal plane surfaces. These experimental investigations have motivated calculations of ethane monolayer structures ${ }^{2,4,5}$ and lattice dynamics ${ }^{5}$ using empirical atom-atom potentials. The calculations have used pairwise-atomic $\mathrm{C}-\mathrm{C}, \mathrm{C}-\mathrm{H}$, and $\mathrm{H}-\mathrm{H}$ potentials of the form $\mathrm{Ar}^{-6}+\mathrm{B} \exp (-\alpha r)$ to represent both intermolecular and molecule-substrate interactions.

Williams and Starr (WS) have noted ${ }^{6}$ that a more accurate representation of the intermolecular interaction in solid hydrocarbons can be obtained by including a long-range Coulomb potential in addition to these short-range potentials. This possibility has interested us in developing an accurate and yet tractable procedure for calculating the intermolecular electrostatic interactions in an ethane monolayer. One method, which we have considered, is to expand the electrostatic potential in terms of point multipoles located at the molecular centers of mass. However, we shall show below that this expansion converges too slowly at typical nearest-neighbor distances in condensed phases to be a practical method of calculation.

An alternative method is to consider the molecular charge distribution to be the superposition of local charge distributions at the atom sites. For hydrocarbons, WS took the simplest of these models in which charges $q_{\mathrm{H}}$ and $q_{\mathrm{C}}$ are assigned to the $\mathrm{H}$ and $\mathrm{C}$ atoms, respectively, subject to the constraint that the molecular monopole and dipole moments vanish. The atom charges were determined by a molecular packing analysis of a variety of hydrocarbon structures. A natural extension of their model is to include higher-order multipoles at each of the atom sites. Buckingham and Fowler $^{7}$ have used atomic-centered multipoles (point charge, dipole, and quadrupole) for calculations of the electrostatic interaction between polar molecules and have re-

\footnotetext{
a) Permanent address: Department of Physics and Astronomy, University of Missouri-Columbia, Columbia, Missouri 65211.
}

ported a good convergence.

In this paper we show that the WS point-charge model and a model which includes higher-order local multipoles give conflicting results for the intramolecular charge distribution of ethane. The point-charge model does not yield a reasonable magnitude nor even the correct sign for the ethane quadrupole moment. On the other hand, the local multipole model yields the negative quadrupole moment observed, with a magnitude close to experimental values. This model also differs from the point-charge model in the sign and magnitude calculated for intermolecular electrostatic interactions at typical nearest-neighbor spacings. Implications of these results for calculating the structure of ethane monolayers are briefly discussed.

We first give a general expression for the molecular quadrupole moment of ethane for a model of point charges at the atom sites. Due to the threefold symmetry of ethane about the $\mathrm{C}-\mathrm{C}$ bond, its quadrupole tensor is diagonal and contains only one independent component, $\theta_{z z}$, where the $z$ axis is taken along the $\mathrm{C}-\mathrm{C}$ bond. The other components are given by $\theta_{x x}=\theta_{y y}=-\frac{1}{2} \theta_{z z}$. Assuming zero values for the molecular monopole moment $\left(q_{c}=-3 q_{\mathrm{H}}\right)$ and dipole moment, the point charge model gives

$$
\theta_{z z}=15.47 \times 10^{-40} q_{H} \mathrm{C} \mathrm{m}^{2}
$$

where $q_{H}$ is the charge on the $\mathrm{H}$ atom in proton units. The standard geometry of the ethane molecule in the staggered configuration $^{4}$ has been assumed with the atomic coordinates as given in Table I. We note that, since the quadrupole moment is the first nonzero moment, its magnitude is independent of the location of the origin.

Since measurements ${ }^{8-10}$ and quantum calculations ${ }^{11-13}$ are in agreement that the ethane quadrupole moment is negative, Eq. (1) has the important consequence that $q_{\mathrm{H}}<0$. This result appears unphysical in that it contradicts the generally accepted view of the charge distribution in the covalent $\mathrm{C}-\mathrm{H}$ bond. ${ }^{14}$ Thus, the atom-site point-charge model is an oversimplification of the molecular charge distribution which, as we show below, yields an erroneous representation of the electric potential about the ethane molecule. 
TABLE 1. The local multipole moments of ethane at the indicated carbon and hydrogen sites as calculated in Ref. 16. The origin is taken at the molecular center of mass, and the unit vector $\hat{z}$ is in the direction of the $\mathrm{C}-\mathrm{C}$ bond. The position coordinates are expressed in angstroms; and the units of the monopole, dipole, and quadrupole moments are $10^{-3} e, 10^{-3} e \AA$, and $10^{-3} e \AA^{2}$, respectively $(e>0)$. The local moments at the other atom sites can be obtained from the appropriate rotation and/or inversion operations.

\begin{tabular}{|c|c|c|c|c|c|}
\hline Atom & \multicolumn{2}{|c|}{$\mathrm{C}$} & \multicolumn{3}{|c|}{$\mathbf{H}$} \\
\hline Position & \multicolumn{2}{|c|}{$0.0 \hat{x}+0.0 \hat{y}+0.771 \hat{z}$} & \multicolumn{3}{|c|}{$0.0 \hat{x}-1.037 \hat{y}+1.137 \hat{z}$} \\
\hline $\begin{array}{l}\text { Monopole } \\
\text { charge, } q_{i}\end{array}$ & \multicolumn{2}{|c|}{-72} & \multicolumn{3}{|c|}{+24} \\
\hline $\begin{array}{c}\text { Dipole } \\
\text { moment, } \mu_{i}\end{array}$ & \multicolumn{2}{|c|}{$0.0 \hat{x}+0.0 \hat{y}+15 \hat{z}$} & \multicolumn{3}{|c|}{$0.0 \hat{x}-67.12 \hat{y}+14.16 \hat{z}$} \\
\hline $\begin{array}{c}\text { Quadrupole } \\
\text { tensor, } \theta_{i}\end{array}$ & $\begin{array}{rr}16.0 & 0.0 \\
0.0 & 16.0 \\
0.0 & 0.0\end{array}$ & $\begin{array}{r}0.0 \\
0.0 \\
-32.0\end{array}$ & $\begin{array}{l}9.0 \\
0.0 \\
0.0\end{array}$ & $\begin{array}{c}0.0 \\
-9.85 \\
10.09\end{array}$ & $\begin{array}{c}0.0 \\
10.09 \\
0.85\end{array}$ \\
\hline
\end{tabular}

A refinement of the point-charge model is to assume a dipole and a quadrupole at the atom sites in addition to a monopole. From the general expression for the $z z$ component of the quadrupole tensor of a charge distribution $\rho(\mathbf{r}),{ }^{15}$

$$
\theta_{z z}=\frac{1}{2} \int d \mathbf{r} \rho(\mathbf{r})\left[3 r_{z}^{2}-r^{2}\right],
$$

it is easy to derive a relation between the molecular quadrupole moment $\theta_{z z}$ and the local charges $q_{i}$, dipole moments $\mu_{i}$, and quadrupole moments $\theta_{i}$. We find

$$
\begin{aligned}
\theta_{z z}= & \frac{1}{2} \sum_{i} q_{i}\left(3 R_{i, z}^{2}-R_{i}^{2}\right) \\
& +\sum_{i}\left(3 R_{i, z} \mu_{i, z}-\mathbf{R}_{i} \cdot \mu_{i}\right)+\sum_{i} \theta_{i, z z},
\end{aligned}
$$

where $\mathbf{R}_{i}$ is the position of the $i$ th atom in the molecule and $R_{i, z}$ is its $z$ component. The large number of parameters in Eq. (3) makes it difficult to find a unique combination of $q_{i}$, $\boldsymbol{\mu}_{i}$, and $\boldsymbol{\theta}_{i}$ which gives the negative quadrupole moment observed for ethane as well as a physically reasonable charge distribution along the $\mathrm{C}-\mathrm{H}$ bonds.

Rather than trying to infer the local multipoles from the measured quadrupole moment, we have used the results of quantum mechanical calculations of the ethane charge distribution to evaluate the quadrupole moment from Eq. (3). Eisenstein and Hirshfeld ${ }^{16}$ have calculated local multipoles in ethane by applying the stockholder recipe ${ }^{17}$ and find a positive charge on hydrogen and a negative charge on carbon in accordance with our expectations. Using their values for the local monopole, dipole, and quadrupole moments as listed in Table I, we calculate from Eq. (3) a molecular quadrupole moment of $-3.43 \times 10^{-40} \mathrm{C} \mathrm{m}^{2}$. This has the same sign and is within $2 \%$ of the latest experimental value ${ }^{10}$ and within $20 \%$ of the latest quantum calculations. ${ }^{13}$ Eisenstein and Hirshfeld emphasize that only qualitative agreement should be expected since they have used a double zeta basis set without polarization functions. Extending the basis set may improve the agreement.

It is instructive to consider the relative magnitude of the three terms in the expression Eq. (3) for the molecular quadrupole moment. They are, in order, $0.37,-2.86$, and -0.94 in units of $10^{-40} \mathrm{C} \mathrm{m}^{2}$. Therefore, the principal contribution to the quadrupole moment comes from the local dipoles rather than from atom-centered charge transfer along the $\mathbf{C}-\mathbf{H}$ bonds. This provides some insight into the failure of the simple point-charge model for ethane.
The point-charge and local multipole models can also be compared conveniently by plotting the difference in the electric potential calculated from the two models in the region surrounding a molecule. In Fig. 1, contours of constant potential difference are plotted for two different planes through a molecule. In the point-charge model, the charges $\left(q_{\mathrm{H}}=-0.22 e\right.$ and $\left.q_{\mathrm{C}}=0.66 e\right)$ have been calculated from Eq. (1) to give the same molecular quadrupole moment as in the multipole model. As expected, the two potentials are nearly equal at large distances, while at typical intermolecular separations in monolayer ethane $(\sim 4.3 \AA)$ their difference is significant.

To explore further the differences between the two models, we have used each to calculate the interaction energy between two ethane molecules in a nested configuration with parallel $\mathrm{C}-\mathrm{C}$ bonds as shown in Fig. 2. This configuration is believed to occur in a high-density monolayer phase of ethane adsorbed on graphite. ${ }^{1-3}$ The ethane monolayer forms a $\sqrt{3} \times \sqrt{3}$ superlattice on the graphite basal planes with the $\mathrm{C}-\mathrm{C}$ bond perpendicular to the surface. The distance between centers of mass of nearest-neighbor molecules is $4.26 \AA$. For the point-charge model, we again take the parameters which give the same molecular quadrupole moment as the local multipole model of Eisenstein and Hirshfeld. ${ }^{16}$ It is important to note that their model assumes point multipoles, since the local charge separations are small compared to the interatomic distances. This assumption greatly simplifies the calculation of the electrostatic potential energy.

Figure 3 shows the potential energy of two neighboring ethane molecules in the monolayer structure of Fig. 2. The energy is plotted as a function of the distance between molecular centers of mass. The most interesting feature of these results is that, at the observed molecular separation of $4.3 \AA$, the models do not even agree on the sign of the interaction. For the point-charge model, the interaction is repulsive, whereas it is attractive for the local multipole model. This conclusion holds regardless of the magnitude of the charges in the point-charge model. As in the case of the molecular quadrupole moment, it is the local dipole contribution which is primarily responsible for the qualitative difference in the interaction energy calculated from the two models.

Finally, we have investigated the feasibility of calculating the electrostatic energy of the pair of ethane molecules in Fig. 2 by an expansion in the multipole moments of the entire molecule. As described in the Appendix, we have used both 

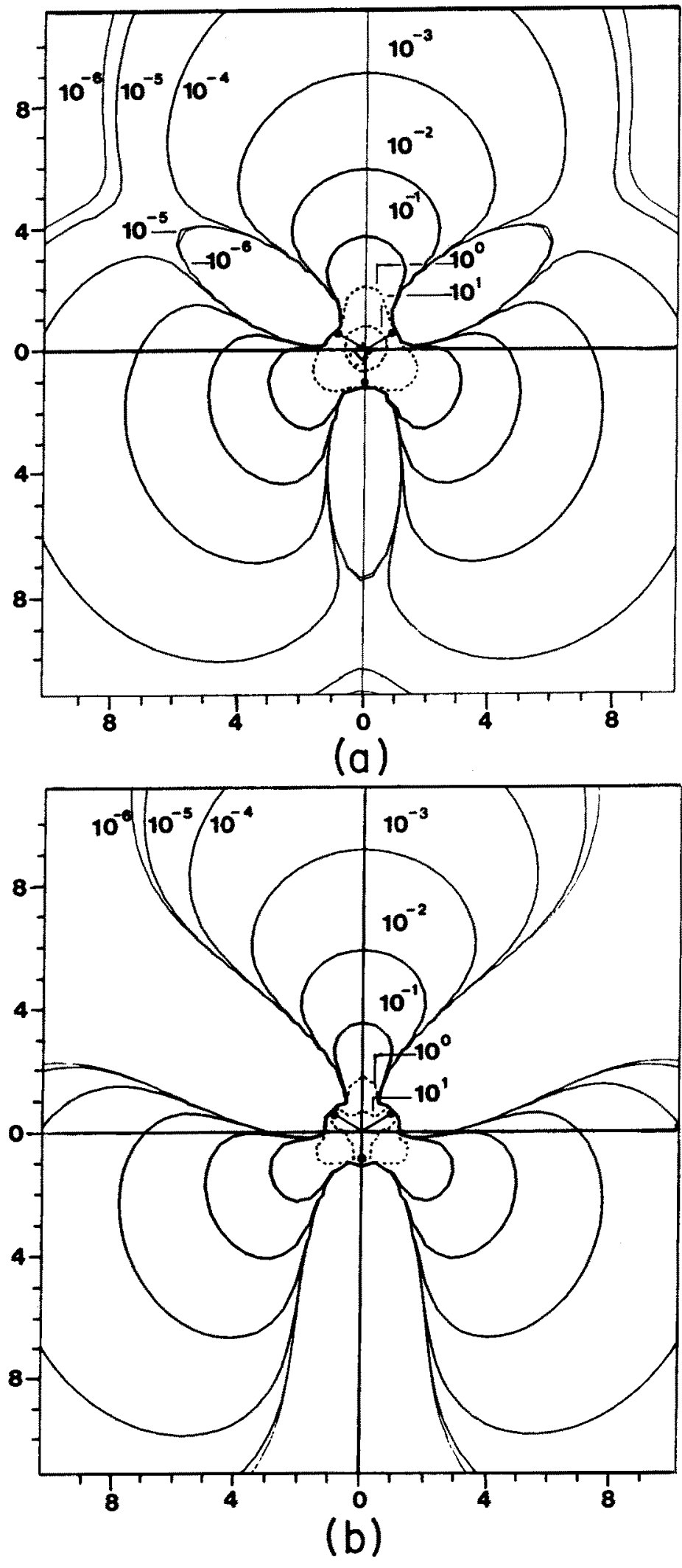

FIG. 1. Contour plots of the difference in the electric potential of an ethane molecule calculated from the atom-site multipole representation of the electric charge distribution and from the atom-site monopole representation: (a) a plane through the upper $\mathrm{C}$ atom and perpendicular to the $\mathrm{C}-\mathrm{C}$ bond; and (b) a plane through the upper $\mathrm{H}$ atoms and perpendicular to the $\mathrm{C}-\mathrm{C}$ bond. The charges in the monopole representation have been adjusted to give the same molecular quadrupole moment as in the local multipole model. The contours are labeled in volts and spatial axes are in units of angstroms. Black circles near the center of each plot indicate the proton positions (b) and their projection on the plane (a).

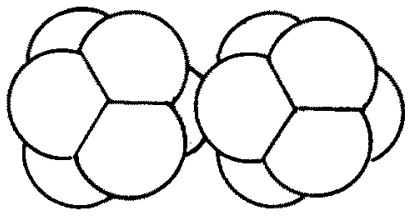

(a)

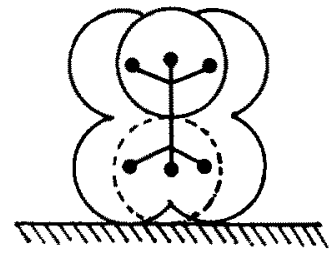

(b)

FIG. 2. Two ethane molecules with parallel $\mathrm{C}-\mathrm{C}$ bonds in a nested configuration as in the high-density monolayer phase on graphite: (a) projection onto the graphite basal plane surface; (b) side view.

the point-charge and local multipole models to calculate the first two nonzero terms in the expansion. We find the ratio of the higher-order quadrupole-hexadecapole term to the quadrupole-quadrupole term to be 1.75 and -1.44 for the point-charge and local multipole models, respectively, at a molecular separation of $4.26 \AA$. This contrasts with the case of monolayers of simple diatomic molecules ${ }^{18}$ where the quadrupole-hexadecapole term in the intermolecular electrostatic energy is only a few percent of the quadrupolequadrupole term. Thus, the first two nonvanishing total moments of the ethane charge distribution are insufficient for calculating the electrostatic interactions of the molecule in condensed phases. This conclusion is in agreement with that of Brobjer and Murrell ${ }^{19}$ for interactions of small polar molecules.

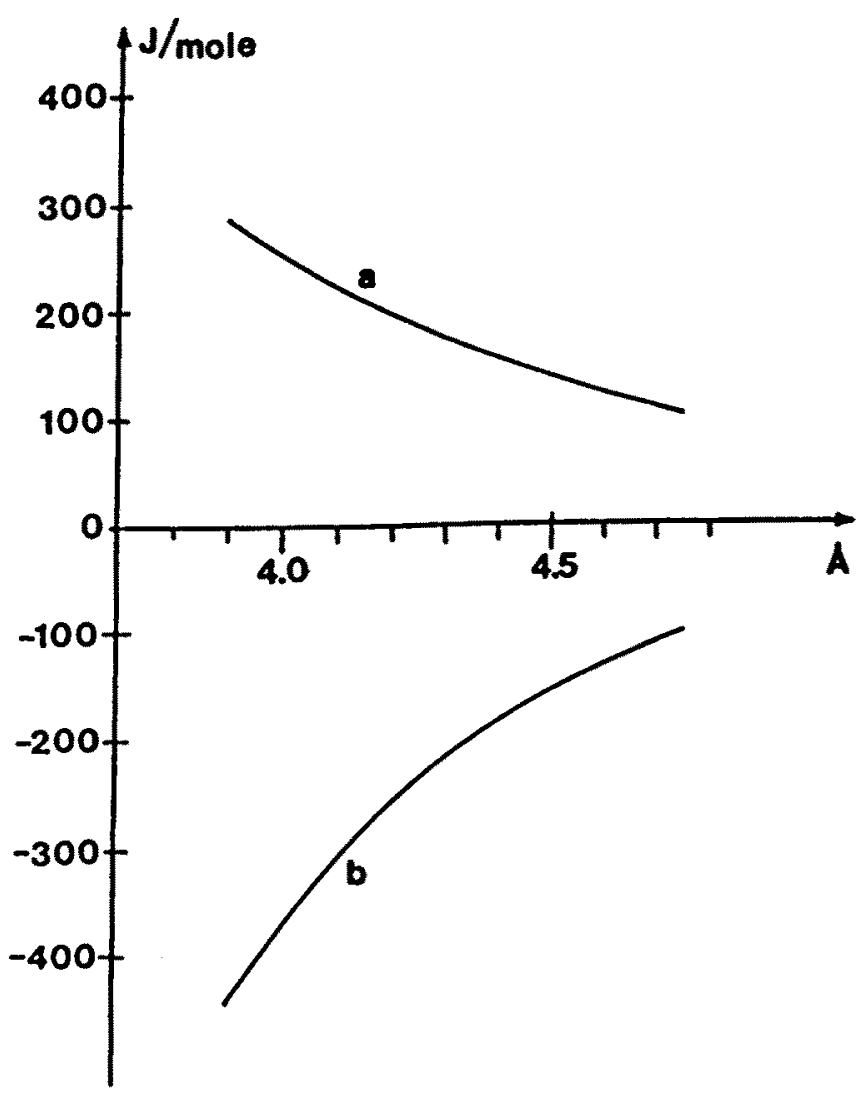

FIG. 3. Electrostatic energy of two ethane molecules in the configuration of Fig. 2 as a function of the distance between the molecular centers of mass: (a) atom-site monopole model; (b) atom-site multipole model. For comparison, the molecular quadrupole-quadrupole energy is $101.5 \mathrm{~J} / \mathrm{mol}$ at a separation of $4.26 \AA$. 
In summary, we have seen that an accurate representation of the charge distribution in ethane requires higher-order moments, particularly dipoles, at the atom sites. This result may have important implications for the derivation of transferable pairwise atomic potentials from a molecular packing analysis. Including higher-order multipole terms in an electrostatic contribution to these potentials could result in a significant renormalization of the short-range interaction parameters. To investigate this possibility, it will be necessary to determine whether the problems encountered with a local monopole representation of the ethane charge distribution are present for other hydrocarbons. This will require detailed quantum calculations of local charge distributions for a number of molecules.

\section{ACKNOWLEDGMENTS}

This work was partially supported by the Danish Natural Science Foundation, the Otto Mønsteds Fond, and National Science Foundation Grants No. DMR-8214518 and No. DMR-8304366.

\section{APPENDIX: MOLECULAR MULTIPOLE EXPANSION OF THE ELECTROSTATIC INTERACTION BETWEEN TWO ETHANE MOLECULES}

We calculate here the quadrupole-quadrupole and quadrupole-hexadecapole terms in the multipole expansion of the electrostatic energy for the pair of molecules in Fig. 2. In general, for quadrupoles $\theta(i)$ and $\theta(j)$ separated by a distance $R$ with the unit vector $\hat{\mathbf{R}}$ directed from $i$ to $j$, the interaction energy is

$$
\begin{aligned}
\phi[\theta(i), \theta(j)]= & \frac{1}{3 R^{5}}\{35[\hat{\mathbf{R}} \cdot \theta(i) \cdot \hat{\mathbf{R}}][\hat{\mathbf{R}} \cdot \theta(j) \cdot \hat{\mathbf{R}}] \\
& -20[\hat{\mathbf{R}} \cdot \theta(i)] \cdot[\hat{\mathbf{R}} \cdot \theta(j)]+2 \theta(i) \cdot \theta(j)\} .
\end{aligned}
$$

In the same notation, the interaction energy of a quadrupole $\boldsymbol{\theta}(i)$ with a hexadecapole $\Phi(j)$ is

$$
\begin{aligned}
\phi & {[\theta(i), \Phi(j)] } \\
= & \left(1 / R^{7} \sum_{\alpha \beta \gamma \delta}\right) \Phi_{\alpha \beta \gamma \delta}(j)\left\{33 \hat{R}_{\alpha} \hat{R}_{\beta} \hat{R}_{\gamma} \hat{R}_{\delta}[\hat{\mathbf{R}} \cdot \theta(i) \cdot \hat{\mathbf{R}}]\right. \\
& \left.-24 \hat{R}_{\alpha} \hat{R}_{\beta} \hat{R}_{\gamma}[\hat{\mathbf{R}} \cdot \theta(i)]_{\delta}+4 \hat{R}_{\alpha} \hat{R}_{\beta} \theta_{\gamma \delta}(i)\right\} .
\end{aligned}
$$

These expressions can be simplified for the nearestneighbor configuration of Fig. 2. Taking $\hat{\mathbf{R}}$ along the $y$ axis and the ethane $\mathrm{C}-\mathrm{C}$ bond in the $z$ direction (normal to the surface), the quadrupole-quadrupole energy of Eq. (A1) reduces to

$$
\phi[\theta(i), \theta(j)]=\frac{9}{4}\left(1 / R^{5}\right) \theta^{2},
$$

where $\theta=\theta_{z z}(i)=\theta_{z z}(j)$.

To evaluate the quadrupole-hexadecapole energy in Eq. (A2), we first note that the symmetry of the ethane molecule allows the components of the hexadecapole tensor to be expressed in terms of three independent parameters, $\Phi=\Phi_{z z z z}, \phi_{x}=\Phi_{x x x z}$, and $\phi_{y}=\Phi_{y y y z}$. The elements of the hexadecapole tensor are listed in Table II where it has been assumed that the origin is at the molecular center of mass.
TABLE II. Representative elements of the hexadecapole tensor for ethane in terms of the three independent parameters $\Phi, \phi_{x}$ and $\phi_{y}$. The tensor has a total of 81 elements. Those corresponding to a permutation of the $x, y$, and $z$ subscripts (e.g., $\Phi_{x^{z}, y^{y}}$ and $\Phi_{x y x y}$ ) are equal. The $z$ axis has been taken along the $\mathrm{C}-\mathrm{C}$ bond.

\begin{tabular}{ccc}
\hline \hline$\Phi_{z^{4}}=\Phi$ & $\Phi_{x^{2}, y^{2}}=1 \Phi$ & $\Phi_{x y, z^{2}}=0$ \\
$\Phi_{y^{4}}=\frac{1}{1} \Phi$ & $\Phi_{x^{3}, z}=\phi_{x}$ & $\Phi_{y, z^{3}}=0$ \\
$\Phi_{x^{4}}=\frac{1}{}$ & $\Phi_{x z, y^{2}}=-\phi_{x}$ & $\Phi_{x^{3}, y}=0$ \\
$\Phi_{x^{2}, z^{2}}=-\frac{1}{2} \Phi$ & $\Phi_{y^{3}, z}=\phi_{y}$ & $\Phi_{y^{3}, x}=0$ \\
$\Phi_{y^{2}, z^{2}}=-\frac{1}{2} \Phi$ & $\Phi_{y z, x^{2}}=-\phi_{y}$ & $\Phi_{x, z^{3}}=0$ \\
\hline \hline
\end{tabular}

Secondly, we note that for the particular configuration in Fig. 2 only the parameter $\Phi$ enters the expression for the quadrupole-hexadecapole energy. We find that Eq. (A2) reduces to

$$
\phi[\theta(i), \phi(j)]=\frac{-75}{16} \frac{1}{R^{7}} \theta \Phi .
$$

Combining Eqs. (A3) and (A4), the ratio of the quadrupole-hexadecapole to the quadrupole-quadrupole energy is

$$
\frac{\phi(\theta, \Phi)}{\phi(\theta, \theta)}=\frac{-25}{12} \frac{1}{R^{2}} \frac{\Phi}{\theta} .
$$

For the simple point-charge model of the molecule, we find

$$
\Phi=-2.32 \times 10^{-58} q_{\mathrm{H}} \mathrm{G} \mathrm{m}^{4},
$$

where $q_{\mathrm{H}}$ is in electron units. Using the expression for $\theta$ from Eq. (1) and assuming a molecular separation $R=4.26 \AA$ the ratio in $\mathrm{Eq}$. (A5) is 1.75 .

To demonstrate that the poor convergence of the molecular multipole expansion is not an artifact of the point charge model, we have also calculated the ratio in Eq. (A5) from the local multipole model of Ref. 16. Using the same notation as in Eq. (3), we can express the hexadecapole parameter $\Phi=\Phi_{z z z z}$ in terms of the local monopole, dipole, and quadrupole moments as

$$
\begin{aligned}
\Phi_{z z z z}= & \frac{1}{8} \sum_{j} q_{j}\left(35 R_{j z}^{4}-30 R_{j}^{2} R_{j z}^{2}+3 R_{j}^{4}\right) \\
& +\frac{1}{8} \sum_{j}\left[140 R_{j z}^{3} \mu_{j z}-60 R_{j}^{2} R_{j z} \mu_{j z}\right. \\
& \left.+\left(12 R_{j}^{2}-60 R_{j z}^{2}\right)\left(\mathbf{R}_{j} \cdot \mu_{j}\right)\right] \\
& +\frac{1}{8} \sum_{j}\left[\left(140 R_{j z}^{2}-20 R_{j}^{2}\right) \theta_{j, z z}\right. \\
& \left.-80 R_{j z}\left(\theta_{j} \cdot \mathbf{R}_{j}\right)_{z}+8\left(\mathbf{R}_{j} \cdot \boldsymbol{\theta}_{j} \cdot \mathbf{R}_{j}\right)\right]
\end{aligned}
$$

Substituting the values of the local moments from Table I into Eq. (A7), gives $\Phi=-42.9 \times 10^{-60} \mathrm{C} \mathrm{m}^{4}{ }^{20}$ The magnitudes of the three terms in Eq. (A7) are, in order, - 5.58, -42.21 , and 4.90 in units of $10^{-60} \mathrm{C} \mathrm{m}^{4}$ so that, as in the case of the molecular quadrupole moment, it is the local dipoles which provide the dominant contribution. Amos and Williams ${ }^{13}$ also calculated $\Phi$ for ethane and found the value $-24.9 \times 10^{-60} \mathrm{C} \mathrm{m}^{4}$ in fair agreement with our result for the atom-site multipole model. With the value of $\theta=-3.43 \times 10^{-40} \mathrm{C} \mathrm{m}^{2}$ computed from Eq. (3), the local multipole model gives -1.44 for the ratio in Eq. (A5) at $R=4.26 \AA$. 
${ }^{1}$ J. P. Coulomb, J. P. Biberian, J. Suzanne, A. Thomy, G. J. Trott, H. Taub, H. R. Danner, and F. Y. Hansen, Phys. Rev. Lett. 43, 1978 (1979); H. Taub, G. J. Trott, F. Y. Hansen, H. R. Danner, J. P. Coulomb, J. P. Biberian, J. Suzanne, and A. Thomy, in Ordering in Two Dimensions, edited by S. K. Sinha (North-Holland, New York, 1980), p. 97.

${ }^{2}$ G. J. Trott, Ph.D. thesis, University of Missouri-Columbia, 1981.

${ }^{3}$ J. Suzanne, J. L. Seguin, H. Taub, and J. P. Biberian, Surf. Sci. 125, 153 (1983).

${ }^{4}$ F. Y. Hansen and H. Taub, Phys. Rev. B 19, 6542 (1979).

${ }^{5}$ F. Y. Hansen, R. Wang, H. Taub, H. Shechter, D. G. Reichel, H. R. Danner, and G. P. Alldredge, Phys. Rev. Lett. 53, 572 (1984).

${ }^{6}$ D. E. Williams and T. L. Starr, Comput. Chem. 1, 173 (1977).

${ }^{7}$ A. D. Buckingham and P. W. Fowler, J. Chem. Phys. 79, 6426 (1983).

${ }^{8}$ D. E. Stogryn and A. P. Stogryn, Mol. Phys. 11, 371 (1966).

${ }^{9}$ A. D. Buckingham, R. L. Disch, and D. A. Dunmer, J. Am. Chem. Soc. 90, 3104 (1968).

${ }^{10}$ A. D. Buckingham, C. Graham, and J. H. Williams, Mol. Phys. 49, 703
(1983).

${ }^{11}$ L. C. Snyder and H. Basch, Molecular Wavefunctions and Properties (Wiley-Interscience, New York, 1972).

12J. D. Goddard and I. G. Cizmadia, Int. J. Quantum Chem. 12, 138 (1977). ${ }^{13}$ R. D. Amos and J. H. Williams, Chem. Phys. Lett. 66, 471 (1979).

${ }^{14}$ R. T. Sanderson, Polar Covalence (Academic, New York, 1983).

15. D. Jackson, Classical Electrodynamics (Wiley, New York, 1962).

${ }^{16} \mathrm{M}$. Eisenstein and F. L. Hirshfeld, Acta Crystallogr. Sect. B 39, 61 (1983).

${ }^{17}$ F. L. Hirshfeld, Theor. Chem. Acta 44, 129 (1977).

${ }^{18}$ L. W. Bruch, J. Chem. Phys. 79, 3148 (1983).

${ }^{19}$ J. T. Brobjer and J. N. Murrell, J. Chem. Soc. Faraday Trans. 278,1853 (1982).

${ }^{20}$ Although not needed in the calculation of the quadrupole-hexadecapole energy for the particular structure in Fig. 2, we have also evaluated the hexadecapole parameters $\phi_{x}$ and $\phi_{y}$ for the ethane molecule in the local multipole model of Ref. 16. We find $\phi_{x}=0$ and $\phi_{y}=-23.7 \times 10^{-60}$ $\mathrm{C} \mathrm{m}^{4}$. 